\title{
Doi-Hopf Modules over Weak Hopf Algebras
}

\author{
Gabriella Böhm ${ }^{1}$ \\ Research Institute for Particle and Nuclear Physics, Budapest \\ H-1525 Budapest 114, P.O.Box 49, Hungary
}

\begin{abstract}
The theory of Doi-Hopf modules [7, 10] is generalized to Weak Hopf Algebras [1, 12, 21.
\end{abstract}

\section{Introduction}

The category ${ }^{C} \mathcal{M}(H)_{A}$ of Doi-Hopf Modules over the bialgebra $H$ was introduced in [7] and independently in [10]. It is the category of the modules over the algebra $A$ which are also comodules over the coalgebra $C$ and satisfy certain compatibility condition involving $H$. The study of ${ }^{C} \mathcal{M}(H)_{A}$ turned out to be very useful: It was shown in [7, 4] that many categories investigated independently before - such as the module and comodule categories over bialgebras, the Hopf modules category [15], and the Yetter-Drinfeld category [16, 14] - are special cases of ${ }^{C} \mathcal{M}(H)_{A}$. Using this observation many results known for module categories over bialgebras or Hopf algebras were generalized to this more general setting [5, 6].

In this paper we generalize the definition of Doi-Hopf modules to the case when $H$ is a Weak Bialgebra (WBA). Our definitions are supported by the fact that many results of [10, 5, 6] remain valid in this case.

Weak Bialgebras (Weak Hopf Algebras - WHA's -) are generalizations of bialgebras (Hopf algebras) see [1, 2] and [12] (latter one using somewhat different terminology). In contrast to another direction of generalization, the quasi-Hopf algebras and weak quasi-Hopf algebras, WBA's are coassociative. Though their counit is not an algebra map, their structure is designed such a way that their (left or right) (co-) module category carries a monoidal structure [12, 3] (and some more in the WHA case [3]).

WHA's have relevance for example in describing depth 2 reducible inclusions 13 .

\footnotetext{
${ }^{1}$ e-mail: BGABR@rmki.kfki.hu

Supported by the Hungarian Scientific Research Fund, OTKA - T 016233
} 
As the bialgebra (Hopf algebra) also the WBA (WHA) is a self-dual structure: The dual space of a finite dimensional WBA (WHA) carries naturally a WBA (WHA) structure [1, 2].

The paper is organized as follows: we define and examine the structures such as the weak Doi-Hopf datum (generalizing the Doi-Hopf datum of [7]) the weak Doi-Hopf module (generalizing the Doi-Hopf module of [7]) the weak smash product (generalizing the analogous notion of [10]) and the weak Doi-Hopf integral (generalizing definitions of [6, 8]). We illustrate these notions on the same four examples generalizing some classical examples of [0, 那.

\section{The Weak Doi-Hopf Datum}

In this Section $H$ is a Weak Bialgebra (WBA) in the sense of [2] over the field $k$. Its unit element is denoted by $\mathbb{1}$, the product of the elements $g, h \in H$ by $g h$, the coproduct of $h \in H$ by $\Delta(h)=h_{(1)} \otimes h_{(2)}$ and the counit is denoted by $\varepsilon$.

Definition 2.1 Let $H$ be a WBA over the field $k$. The $k$-algebra $A$ is a left $H$ comodule algebra if there exists a left weak coaction $\rho$ of $H$ on $A$ which is also an algebra map. I.e. a map $\rho: A \rightarrow H \otimes A$ such that

$$
\begin{aligned}
\left(\operatorname{id}_{H} \otimes \rho\right) \circ \rho & =\left(\Delta \otimes \operatorname{id}_{A}\right) \circ \rho \\
(\mathbb{1} \otimes a) \rho\left(1_{A}\right) & =\left(\Pi^{R} \otimes \operatorname{id}_{A}\right) \circ \rho(a) \\
\rho(a b) & =\rho(a) \rho(b)
\end{aligned}
$$

for all $a, b \in A$. We use the standard notation $\rho(a)=a_{<-1>} \otimes a_{<0>}$ and $\left(\Delta \otimes \mathrm{id}_{A}\right) \circ$ $\rho(a)=a_{<-2>} \otimes a_{<-1>} \otimes a_{<0>}=\left(\operatorname{id}_{H} \otimes \rho\right) \circ \rho(a)$.

The left weak coaction $\rho$ is non-degenerate if $\left(\varepsilon \otimes \mathrm{id}_{A}\right) \circ \rho=\mathrm{id}_{A}$ or, equivalently, $\left(\varepsilon \otimes \mathrm{id}_{A}\right) \circ \rho\left(1_{A}\right)=1_{A}$. For non-degenerate left weak coactions $\rho$ (2.1b) has an equivalent form (compare with [13) $\left(\Delta \otimes \mathrm{id}_{A}\right) \circ \rho\left(1_{A}\right)=\left(\mathbb{1} \otimes \rho\left(1_{A}\right)\right)\left(\Delta(\mathbb{1}) \otimes 1_{A}\right)$.

Similarly, $A$ is a right $H$-comodule algebra if there exists a right weak coaction $\rho$ of $H$ on $A$ which is also an algebra map. I.e. a map $\rho: A \rightarrow A \otimes H$ such that

$$
\begin{aligned}
\left(\rho \otimes \mathrm{id}_{H}\right) \circ \rho & =\left(\operatorname{id}_{A} \otimes \Delta\right) \circ \rho \\
\rho\left(1_{A}\right)(a \otimes \mathbb{1}) & =\left(\operatorname{id}_{A} \otimes \Pi^{L}\right) \circ \rho(a) \\
\rho(a b) & =\rho(a) \rho(b)
\end{aligned}
$$

for all $a, b \in A$. We also denote $\rho(a)=a_{<0>} \otimes a_{<1>}$.

The right weak coaction $\rho$ is non-degenerate if $\left(\mathrm{id}_{A} \otimes \varepsilon\right) \circ \rho=\mathrm{id}_{A}$ or, equivalently, if $\left(\mathrm{id}_{A} \otimes \varepsilon\right) \circ \rho\left(1_{A}\right)=1_{A}$. For non-degenerate right weak coactions $\rho$ (2.2b) has an equivalent form $\left(\operatorname{id}_{A} \otimes \Delta\right) \circ \rho\left(1_{A}\right)=\left(1_{A} \otimes \Delta(\mathbb{1})\right)\left(\rho\left(1_{A}\right) \otimes \mathbb{1}\right)$.

The dual notion to comodule algebra is the module coalgebra defined as follows: The $k$-coalgebra $C$ is a right $H$-module coalgebra if there exists a right weak action of $H$ on $C$ which is also a coalgebra map. I.e. a map $: C \times H \rightarrow C$ such that

$$
\begin{aligned}
(c \cdot g) \cdot h & =c \cdot(g h) \\
c \cdot \Pi^{L}(h) & =\varepsilon_{C}\left(c_{(1)} \cdot h\right) c_{(2)} \\
\Delta_{C}(c \cdot h) & =\Delta_{C}(c) \cdot \Delta(h)
\end{aligned}
$$


for all $c \in C, g, h \in H$.

The right weak action - is non-degenerate if $c \cdot \mathbb{1}=c \forall c \in C$ or, equivalently, if $\varepsilon_{C}(c \cdot \mathbb{1})=\varepsilon_{C}(c) \forall c \in C$. For non-degenerate right weak actions $\cdot$ (2.3b) has the equivalent reformulation as $\varepsilon_{C}(c \cdot h)=\varepsilon\left(c \cdot \Pi^{L}(h)\right)$.

Similarly, $C$ is a left $H$-module coalgebra if there exists a left weak action of $H$ on $C$ which is also a coalgebra map. I.e. a map $: H \times C \rightarrow C$ such that

$$
\begin{aligned}
g \cdot(h \cdot c) & =(g h) \cdot c \\
\Pi^{R}(h) \cdot c & =c_{(1)} \varepsilon_{C}\left(h \cdot c_{(2)}\right) \\
\Delta_{C}(h \cdot c) & =\Delta(h) \cdot \Delta_{C}(c)
\end{aligned}
$$

for all $c \in C g, h \in H$.

The left weak action - is non-degenerate if $\mathbb{1} \cdot c=c \forall c \in C$ or, equivalently, if $\varepsilon_{C}(\mathbb{1} \cdot c)=\varepsilon_{C}(c) \forall c \in C$. For non-degenerate left weak actions $\cdot$ (2.4b) has the equivalent reformulation $\varepsilon_{C}(h \cdot c)=\varepsilon\left(\Pi^{R}(h) \cdot c\right)$.

Notice, that in contrast to the case when $H$ is an ordinary bialgebra the unit preserving property of $\rho$ and the counit preserving property of - are not required and the form of condition (b) in each group is somewhat different from the usual one.

Definition 2.2 $A$ right Weak Doi-Hopf datum is a triple $(H, A, C)$, where $H$ is a $W B A$ over $k, A$ a left $H$-comodule algebra and $C$ a right $H$-module coalgebra.

$A$ left Weak Doi-Hopf datum is a triple $(H, A, C)$ where $H$ is a WBA over $k, A$ a right $H$-comodule algebra and $C$ a left $H$-module coalgebra.

$A$ (left or right) Weak Doi-Hopf datum is non-degenerate if both the weak coaction of $H$ on $A$ and the weak action of $H$ on $C$ are non-degenerate.

\section{Examples:}

1 Let $H$ be a WBA over $k, A:=H$ as an algebra with the coaction $\rho:=\Delta$, $C:=H^{L}$ with the coalgebra structure

$$
\begin{aligned}
\Delta_{H^{L}}\left(a^{L}\right): & =\mathbb{1}_{(2)} a^{L} \otimes S\left(\mathbb{1}_{(1)}\right) \equiv \mathbb{1}_{(2)} \otimes a^{L} S\left(\mathbb{1}_{(1)}\right) \\
\varepsilon_{H^{L}}\left(a^{L}\right): & =\varepsilon\left(a^{L}\right)
\end{aligned}
$$

and the action $a^{L} \cdot h:=\mathbb{1}_{(2)} \varepsilon\left(a^{L} h \mathbb{1}_{(1)}\right)$ for all $a^{L} \in H^{L}, h \in H$. Then $(H, A=$ $\left.H, C=H^{L}\right)$ is a non-degenerate right Weak Doi-Hopf datum.

2 Let $H$ be a WBA over $k, A:=H^{L}$ as the subalgebra of $H$ with the coaction $\rho:=\left.\Delta\right|_{H^{L}}, C:=H$ as a coalgebra with the action $c \cdot h:=c h$ for all $c, h \in H$. Then $\left(H, A=H^{L}, C=H\right)$ is a non-degenerate right Weak Doi-Hopf datum.

3 Let $H$ be a WBA over $k, A:=H$ as an algebra with the coaction $\rho:=\Delta$, $C:=H$ as a coalgebra with the action $c \cdot h=c h$ for all $c, h \in H$. Then $(H, A=$ $H, C=H)$ is a non-degenerate right Weak Doi-Hopf datum.

4 Let $K$ be a WHA over $k, H:=K^{o p} \otimes K$ as a bialgebra. ( $K^{o p}$ is the bialgebra with the same coalgebra structure as $K$ and the opposite algebra structure.) $A:=K$ as an algebra with the coaction $\rho(a):=\left(S^{-1}\left(a_{(3)}\right) \otimes a_{(1)}\right) \otimes a_{(2)}$ for all $a \in K, C:=K$ 
as a coalgebra with the action $c \cdot(a \otimes b):=a c b$ for all $c \in K,(a \otimes b) \in H$. Then ( $\left.H=K^{o p} \otimes K, A=K, C=K\right)$ is a non-degenerate right weak Doi-Hopf datum.

Let us call a (left or right) weak Doi-Hopf datum finite dimensional if all $H, A$ and $C$ are finite dimensional as $k$-spaces. There is a well defined notion of duality for finite dimensional weak Doi- Hopf data sending a left weak Doi-Hopf datum to a right one and vice versa:

Introduce the following notations: For any finite dimensional $k$ - space $M$ let $\hat{M}$ denote the dual $k$-space. If $A$ is a finite dimensional algebra then by $\hat{A}$ we mean the dual space equipped with the dual coalgebra structure. Similarly, for a finite dimensional coalgebra $C$ denote the dual algebra by $\hat{C}$ and finally for a finite dimensional bialgebra $H$ denote the dual bialgebra by $\hat{H}$.

Proposition 2.3 For a (non-degenerate) right weak Doi-Hopf datum $(H, A, C)$ the triple $(\hat{H}, \hat{C}, \hat{A})$ is a (non-degenerate) left weak Doi-Hopf datum-called the dual of $(H, A, C)-$ with

$$
\begin{aligned}
& \hat{\rho}(\hat{c}):=b_{i} \triangleright \hat{c} \otimes \beta^{i} \\
& \phi \cdot \hat{a}:=(\phi \otimes \hat{a}) \circ \rho
\end{aligned}
$$

where $\hat{c} \in \hat{C},\left\{b_{i}\right\}$ is any basis in $H$ and $\left\{\beta^{i}\right\}$ is the dual basis in $\hat{H}, \phi \in \hat{H}, \hat{a} \in \hat{A}$ and $(h \triangleright \hat{c})(d)=\hat{c}(d \cdot h)$ for $\hat{c} \in \hat{C}, d \in C, h \in H$.

Similarly, for a (non-degenerate) left weak Doi-Hopf datum $(H, A, C)$ the triple $(\hat{H}, \hat{C}, \hat{A})$ is a (non-degenerate) right weak Doi-Hopf datum - called the dual of $(H, A, C)-$ with

$$
\begin{aligned}
\hat{\rho}(\hat{c}): & =\beta^{i} \otimes \hat{c} \triangleleft b_{i} \\
\hat{a} \cdot \phi & =(\hat{a} \otimes \phi) \circ \rho
\end{aligned}
$$

with the obvious notation. The above duality transformation is involutive.

Proof: The transformations (2.5) and (2.6) are obviously inverses of one other. One easily checks that (2.2a) for $(\hat{H}, \hat{C}, \hat{A})$ is equivalent to (2.3a) on $(H, A, C),(2.2 \mathrm{~b})$ to $(2.3 \mathrm{~b}), 2.2 \mathrm{~d})$ to $(2.3 \mathrm{~d}),(2.4 \mathrm{a})$ to $(2.1 \mathrm{a}),(2.4 \mathrm{~b})$ to $(2.1 \mathrm{~b})$ and $(2.4 \mathrm{~d})$ to $(2.1 \mathrm{c})$.

The non-degeneracy of the weak coaction $\hat{\rho}$ of $\hat{H}$ on $\hat{C}$ is equivalent to the nondegeneracy of the weak action of $H$ on $C$ while the non-degeneracy of the action of $\hat{H}$ on $\hat{A}$ is equivalent to the non-degeneracy of the weak coaction $\rho$ of $H$ on $A$ both in the left and right cases.

\section{The Weak Doi-Hopf Module}

Definition 3.1 The $k$-space $M$ is a right weak Doi-Hopf module over the right weak Doi-Hopf datum $(H, A, C)$ if it is a non-degenerate right $A$-module and a non-degenerate left $C$-comodule i.e. there exists an action $: M \times A \rightarrow M$ for which $m \cdot 1_{A}=m \forall m \in M$ and a coaction $\rho_{M}: M \rightarrow C \otimes M$ for which $\left(\varepsilon_{C} \otimes \operatorname{id}_{M}\right) \circ \rho_{M}=\rho_{M}$ such that the compatibility condition

$$
\rho_{M}(m \cdot a)=m_{<-1>} \cdot a_{<-1>} \otimes m_{<0>} \cdot a_{<0>}
$$


holds for $\rho_{M}(m) \equiv m_{<-1>} \otimes m_{<0>}$.

Similarly, $M$ is a left weak Doi-Hopf module over the left Doi-Hopf datum $(H, A, C)$ if it is a non-degenerate left $A$-module (with $A$-action •) and a nondegenerate right $C$-comodule (with $C$-coaction $\rho_{M}$ ) such that

$$
\rho_{M}(a \cdot m)=a_{<0>} \cdot m_{<0>} \otimes a_{<1>} \cdot m_{<1>} .
$$

The category ${ }^{C} \mathcal{M}(H)_{A}$ has as objects the finite dimensional right weak Doi-Hopf modules $M$ over the right weak Doi-Hopf datum $(H, A, C)$ and arrows $T: M \rightarrow M^{\prime}$ which intertwine both the $A$-actions and the $C$-coactions:

$$
T(m \cdot a)=T(m) \cdot a \quad \rho_{M^{\prime}} \circ T=\left(\operatorname{id}_{C} \otimes T\right) \circ \rho_{M}
$$

for all $m \in M, a \in A$.

Similarly, ${ }_{A} \mathcal{M}(H)^{C}$ is the category of finite dimensional left weak Doi-Hopf modules over the left Doi-Hopf datum $(H, A, C)$.

Let us see what categories ${ }^{C} \mathcal{M}(H)_{A}$ are in our earlier examples:

\section{Examples:}

$1{ }^{C} \mathcal{M}(H)_{A}$ is equivalent to $\mathcal{M}_{A \equiv H}$, the category of right $H$-modules. The equivalence functor $F:{ }^{C} \mathcal{M}(H)_{A} \rightarrow \mathcal{M}_{A}$ is the forgetful functor.

$2^{C} \mathcal{M}(H)_{A}$ is equivalent to ${ }^{C} \equiv H \mathcal{M}$, the category of left $H$-comodules. The equivalence functor $\hat{F}:{ }^{C} \mathcal{M}(H)_{A} \rightarrow{ }^{C} \mathcal{M}$ is the forgetful functor.

$3^{C} \mathcal{M}(H)_{A}$ is equivalent to ${ }^{H} \mathcal{M}_{H}$, the category of weak Hopf modules [1, 2] over $H$.

$4^{C} \mathcal{M}(H)_{A}$ is equivalent to $\mathcal{Y} \mathcal{D}\left(K_{c o p}^{o p}\right)^{o p}$, the category of (some twisted version of) Yetter-Drinfeld modules over $H$. (For its definition see the Appendix).

Proposition 3.2 Let $(H, A, C)$ be a finite dimensional right weak Doi-Hopf datum and $(\hat{H}, \hat{C}, \hat{A})$ its dual. Then the categories ${ }^{C} \mathcal{M}(H)_{A}$ and $\hat{C}^{\mathcal{M}}(\hat{H})^{\hat{A}}$ are equivalent.

Proof: Let us define the functor $D:{ }^{C} \mathcal{M}(H)_{A} \rightarrow{ }_{\hat{C}} \mathcal{M}(\hat{H})^{\hat{A}}$

$$
\begin{aligned}
D(M):=\hat{M} \text { as a } k-\text { space } & \hat{c} \cdot \mu:=(\hat{c} \otimes \mu) \circ \rho_{M} \\
& \hat{\rho}_{\hat{M}}(\mu):=a_{i} \triangleright \mu \otimes \alpha^{i} \\
D(T):=T^{t} &
\end{aligned}
$$

where $M$ is an object and $T$ an arrow in ${ }^{C} \mathcal{M}(H)_{A},{ }^{t}$ means transposition of linear operators, $\hat{c} \in \hat{C}, \mu \in \hat{M},(a \triangleright \mu)(m)=\mu(m \cdot a)$ for $a \in A, \mu \in \hat{M}, m \in M,\left\{a_{i}\right\}$ is a basis for $A$ and $\left\{\alpha^{i}\right\}$ is the dual basis for $\hat{A}$. One checks by direct calculation that $D$ defines an equivalence functor.

Proposition 3.3 Let $(H, A, C)$ be a non-degenerate right weak Doi-Hopf datum. Then the forgetful functor $F:{ }^{C} \mathcal{M}(H)_{A} \rightarrow \mathcal{M}_{A}$ has a left adjoint and $\hat{F}:{ }^{C} \mathcal{M}(H)_{A} \rightarrow$ ${ }^{C} \mathcal{M}$ has a right adjoint. 
Proof: Our proof is consructive. Define $G: \mathcal{M}_{A} \rightarrow{ }^{C} \mathcal{M}(H)_{A}$ by

$$
\begin{aligned}
G(M):= & C \cdot 1_{A<-1>} \otimes M \cdot 1_{A<0>} \quad \text { as a } k-\text { space } \\
& (c \otimes m) \cdot a:=c \cdot a_{<-1>} \otimes m \cdot a_{<0>} \\
& \rho_{G(M)}:=\left.\left(\Delta_{C} \otimes \operatorname{id}_{M}\right)\right|_{G(M)} \\
G(T):= & \left(i d_{C} \otimes T\right)
\end{aligned}
$$

for $M$ an object and $T$ an arrow in ${ }^{C} \mathcal{M}(H)_{A}, a \in A,(c \otimes m) \in G(M) \subset C \otimes M$.

The fact that $G$ is a left adjoint of $F$ is justified by the existence of unit and counit natural homomorphisms $\rho: \operatorname{id}_{C} \mathcal{M}(H)_{A} \rightarrow G \circ F$ and $\delta: F \circ G \rightarrow \operatorname{id}_{\mathcal{M}_{A}}$. Define them as

$$
\begin{array}{ll}
\rho_{M}: M \rightarrow G(M) & \rho_{M}(m):=m_{<-1>} \otimes m_{<0>} \\
\delta_{M}: G(M) \rightarrow M & \delta_{M}:=\left.\left(\varepsilon_{C} \otimes \operatorname{id}_{M}\right)\right|_{G(M)} .
\end{array}
$$

It is staightforward to show that $\rho_{M} \in(M, G(M))_{C} \mathcal{M}(H)_{A}$, and $\rho$ is natural. The proof of $\delta_{M} \in(G(M), M)_{\mathcal{M}_{A}}$ lies on the following

Lemma 3.4 Let $(H, A, C)$ be a non-degenerate right weak Doi-Hopf datum. Then for any $c \in C$ and $a \in A$

(i) $\quad \Delta_{C}\left(c \cdot 1_{A<-1>}\right) \otimes 1_{A<0>}=c_{(1)} \otimes c_{(2)} \cdot 1_{A<-1>} \otimes 1_{A<0>}$

(ii) $\quad \Pi^{L}\left(a_{<-1>}\right) \otimes a_{<0>}=\Pi^{L}\left(1_{A<-1>}\right) \otimes 1_{A<0>} a$.

Lemma 3.4 (ii) implies $\varepsilon_{C}\left(c \cdot a_{<-1>}\right) a_{<0>}=\varepsilon_{C}\left(c \cdot 1_{A<-1>}\right) 1_{<0>} a$ and hence $\delta_{M} \in(G(M), M)_{\mathcal{M}_{A}}$. Naturality of $\delta$ is obvious.

One can proceed the same way in the case of $\hat{F}$ using now Lemma 3.4 (i). Define $\hat{G}:{ }^{C} \mathcal{M} \rightarrow{ }^{C} \mathcal{M}(H)_{A}$ as

$$
\begin{aligned}
\hat{G}(M):= & \left\{\varepsilon_{C}\left(m_{<-1>} \cdot a_{<-1>}\right) m_{<0>} \otimes a_{<0>} \mid m \in M, a \in A\right\} \text { as a } k-\text { space } \\
& (m \otimes a) \cdot b:=\varepsilon_{C}\left(m_{<-1>} \cdot a_{<-1>} b_{<-1>}\right) m_{<0>} \otimes a_{<0>} b_{<0>} \\
& \rho_{\hat{G}(M)}(m \otimes a):=m_{<-1>} \cdot a_{<-1>} \otimes m_{<0>} \otimes a_{<0>} \\
\hat{G}(T):= & T \otimes \operatorname{id}_{A}
\end{aligned}
$$

for $M$ an object and $T$ an arrow in ${ }^{C} \mathcal{M}(H)_{A},(m \otimes a) \in \hat{G}(M) \subset M \otimes A, b \in A$.

The unit and counit natural homomorphisms $\hat{\rho}: \operatorname{id}_{C} \mathcal{M} \rightarrow \hat{F} \circ \hat{G}$ and $\hat{\delta}: \hat{G} \circ \hat{F} \rightarrow$ $\operatorname{id}_{C} \mathcal{M}(H)_{A}$ can be given by

$$
\begin{array}{ll}
\hat{\rho}_{M}: M \rightarrow \hat{G}(M) & \hat{\rho}_{M}(m):=\varepsilon_{C}\left(m_{<-1>} \cdot 1_{A<-1>}\right) m_{<0>} \otimes 1_{A<0>} \\
\hat{\delta}_{M}: \hat{G}(M) \rightarrow M & \hat{\delta}_{M}(m \otimes a):=m \cdot a
\end{array}
$$




\section{The Weak Smash Product}

Definition 4.1 For the non-degenerate right weak Doi-Hopf datum $(H, A, C)$ define the weak smash product algebra $A \# \hat{C}$ as the $k$-space $1_{A<0>} A \otimes 1_{A<-1>} \triangleright \hat{C}$ equipped with the multiplication rule

$$
(a \# \hat{c})(b \# \hat{d}):=\left(a_{<0>} b \# \hat{c}\left(a_{<-1>} \triangleright \hat{d}\right)\right)
$$

for $(a \# \hat{c}),(b \# \hat{d}) \in A \# \hat{C}$.

One checks that (4.1) makes $A \# \hat{C}$ an associative algebra with unit element $1_{A<0>} \#$ $1_{A<-1>} \triangleright 1_{\hat{C}}$.

Let us see what algebras $A \# \hat{C}$ are in our earlier examples.

\section{Examples:}

$\mathbf{1}(A \equiv H) \#\left(\hat{C} \equiv \hat{H}^{R}\right)$ is isomorphic to $H$, the isomorphism being given by $\iota: A \# \hat{C} \rightarrow H, \iota:=\operatorname{id}_{H} \otimes \varepsilon_{\hat{H}}$.

$2\left(A \equiv H^{L}\right) \#(\hat{C} \equiv \hat{H})$ is isomorphic to $\hat{H}$, the isomorphism being given by $\iota: A \# \hat{C} \rightarrow \hat{H}, \iota:=\varepsilon \otimes \operatorname{id}_{\hat{H}}$.

$3(A \equiv H) \#(\hat{C} \equiv \hat{H})$ is isomorphic to the Weyl algebra or Heisenberg double $\hat{H} \rtimes H$ [1, 2], the isomorphism being given by $\iota: A \# \hat{C} \rightarrow \hat{H} \rtimes H, \iota\left(\mathbb{1}_{(2)} a \#\left(\mathbb{1}_{(1)} \rightarrow\right.\right.$ $\phi)):=\phi a$. (In all of the examples $\rightarrow$ denotes the Sweedler's arrow [15].)

$4(A \equiv K) \#(\hat{C} \equiv \hat{K})$ is isomorphic to the (twisted) Drinfel'd double $\mathcal{D}\left(K_{c o p}^{o p}\right)^{o p}$ (for its definition see the Appendix). The equivalence is given by $\iota: A \# \hat{C} \rightarrow$ $\mathcal{D}\left(K_{c o p}^{o p}\right)^{o p}, \iota\left(\mathbb{1}_{(2)} a \#\left(\mathbb{1}_{(1)} \rightarrow \phi \leftarrow S^{-1}\left(\mathbb{1}_{(3)}\right)\right)\right):=\mathcal{D}(\phi) \mathcal{D}(a)$.

Proposition 4.2 Let $(H, A, C)$ be a non-degenerate right weak Doi-Hopf datum such that $C$ is finite dimensional as a $k$-space. Then the categories ${ }^{C} \mathcal{M}(H)_{A}$ and $\mathcal{M}_{A \# \hat{C}}$ are isomorphic.

Proof: We have the functor $P:{ }^{C} \mathcal{M}(H)_{A} \rightarrow \mathcal{M}_{A \# \hat{C}}$

$$
\begin{gathered}
P(M):=M \text { as a } k-\text { space } \quad m \cdot(a \# \hat{c}):=\hat{c}\left(m_{<-1>}\right) m_{<0>} \cdot a \\
P(T):=T
\end{gathered}
$$

for $M$ an object and $T$ an arrow in ${ }^{C} \mathcal{M}(H)_{A},(a \# \hat{c}) \in A \# \hat{C}, m \in M$.

If $C$ is finite dimensional as a $k$-space then let $\left\{c_{i}\right\}$ be any basis for $C$ and $\left\{\gamma^{i}\right\}$ the dual basis for $\hat{C}$ and construct the inverse functor $P^{\prime}: \mathcal{M}_{A \# \hat{C}} \rightarrow{ }^{C} \mathcal{M}(H)_{A}$ of $P$ :

$$
\begin{aligned}
& P^{\prime}(M):=M \text { as a } k-\text { space } \quad m \cdot a:=m \cdot\left(1_{A<0>} a \# 1_{\left.A<-1>\triangleright 1_{\hat{C}}\right)}\right. \\
& \rho_{M}(m):=c_{i} \otimes m \cdot\left(1_{A<0>} \# 1_{A<-1>} \triangleright \gamma^{i}\right) \\
& P^{\prime}(T):=T
\end{aligned}
$$

for $M$ an object and $T$ an arrow of ${ }^{C} \mathcal{M}(H)_{A}, a \in A, m \in M$. 


\section{Integrals for Weak Doi-Hopf Data}

Let $(H, A, C)$ be a non-degenerate right weak Doi-Hopf datum where $H$ is a weak $H o p f$ algebra with antipode $S, F:{ }^{C} \mathcal{M}(H)_{A} \rightarrow \mathcal{M}_{A}$ the forgetful functor, $G$ its left adjoint as in Proposition 3.3. $V$ be the $k$-space of the natural homomorphisms $\nu: G \circ F \rightarrow \operatorname{id}_{C} \mathcal{M}(H)_{A}$, called the space of integrals for the weak Doi-Hopf datum $(H, A, C)$. We have a straightforward generalization of Theorem 2.3 of [6]:

Theorem 5.1 The space $V$ is isomorphic to the space $V_{4}$ :

$$
\begin{aligned}
V_{4}:=\left\{\gamma: C \rightarrow(C, A)_{\operatorname{Lin}}\right. & \mid \forall c, d \in C \quad a \in A \\
\gamma(c)(d) a & =a_{<0>} \gamma\left(c \cdot a_{<-2>}\right)\left(d \cdot a_{<-1>}\right) \\
c_{(1)} \otimes \gamma\left(c_{(2)}\right)(d) & \left.=d_{(2)} \cdot \gamma(c)\left(d_{(1)}\right)_{<-1>} \otimes \gamma(c)\left(d_{(1)}\right)_{<0>}\right\} .
\end{aligned}
$$

Furthermore the isomorphism $f_{4}: V \rightarrow V_{4}$ takes $\nu \in V$ to a normalized element of $V_{4}$ i.e. to an element $\gamma \in V_{4}$ such that $\gamma\left(c_{(1)}\right)\left(c_{(2)}\right)=\varepsilon_{C}\left(c \cdot 1_{A<-1>}\right) 1_{A<0>}$ if and only if $\nu$ is a splitting of the unit natural homomorphism $\rho: \operatorname{id}_{C} \mathcal{M}(H)_{A} \rightarrow G \circ F$.

The relevance of the existence of normalized elements in $V_{4}$ is discussed in [6].

Let us turn to the investigation of the space of integrals over the weak DoiHopf datum $(H, A, C)$ in our earlier examples. In doing so we make the additional assumption in the Examples $\mathbf{2}$ and $\mathbf{3}$ on $H$ and in $\mathbf{4}$ on $K$ to be a Frobenius WHA. Under this additional condition we identify the space of integrals for the weak DoiHopf datum $(H, A, C)$ with certain subspace of the smash product algebra $A \# \hat{C}$. Also the normalization condition is formulated as a relation in the algebra $A \# \hat{C}$.

In all of the examples $r$ be a non-degenerate right integral in $H$ and $\rho$ the dual right integral [2] in $\hat{H}$.

Examples:

1 The space of Doi-Hopf integrals over $(H, A, C)$ is isomorphic to $V_{0}:=$ Center $H$. Construct the isomorphism $f: V_{4} \rightarrow V_{0}$ as

$$
f(\gamma):=\gamma(\mathbb{1})(\mathbb{1})
$$

The unique normalized element of $V_{0}$ is the unit element $\mathbb{1}$ of $H$.

2 The space of the Doi-Hopf integrals is isomorphic to $V_{0}:=\left(\hat{H}^{R}\right)^{\prime} \cap \hat{H}$, the commutant of the right subalgebra in $\hat{H}$. Let us construct the isomorphism $f$ : $V_{4} \rightarrow V_{0}$ as

$$
[f(\gamma)](h):=\varepsilon(\gamma(r)(h))
$$

for all $h \in H$.

An element $\xi \in V_{0}$ is normalized if

$$
\hat{S}^{-1}\left(\rho_{(2)}\right) \xi \rho_{(1)}=\hat{\mathbb{1}}
$$

holds in $\hat{H}$.

The space $V_{0}$ is not isomorphic to the space $\mathcal{I}^{L}(\hat{H})$ of left integrals in $\hat{H}$. It is its subspace $\hat{H}^{L}$ which is isomorphic to $\mathcal{I}^{L}(\hat{H})$ via the isomorphism $g: \mathcal{I}^{L}(\hat{H}) \rightarrow \hat{H}^{L}$, 
$g(\lambda):=\hat{S}(\lambda<r)$. It is but true that the existence of normalized elements in $\mathcal{I}^{L}(\hat{H})$ and $V_{0}$ are equivalent.

3 The space of the Doi-Hopf integrals is isomorphic to $V_{0}:=H^{\prime} \cap(\hat{H} \rtimes H)$, the commutant of $H$ in the Weyl algebra. The isomorphism $f: V_{4} \rightarrow V_{0}$ is given by

$$
f(\gamma):=\beta^{i} \gamma(r)\left(b_{i}\right)
$$

with the help of the basis $\left\{b_{i}\right\}$ of $H$ and the dual basis $\left\{\beta^{i}\right\}$ of $\hat{H}$.

The element $w \in V_{0}$ is normalized if

$$
\hat{S}^{-1}\left(\rho_{(2)}\right) w \rho_{(1)}=1_{\hat{H} \rtimes H}
$$

holds in the Weyl algebra $\hat{H} \rtimes H$.

4 The space of the Doi-Hopf integrals is isomorphic to $V_{0}:=\left\{u \in \mathcal{D}\left(K_{c o p}^{o p}\right)^{o p} \mid\right.$ $\left.u \mathcal{D}(b)=\mathcal{D}\left(b_{(1)}\right) u \mathcal{D}\left(S^{-1}(r) S^{-2}\left(b_{(2)}\right) \rightarrow \rho\right)\right\}$. The isomorphism $f: V_{4} \rightarrow V_{0}$ is given by

$$
f(\gamma):=\mathcal{D}\left(\beta^{i}\right) \mathcal{D}\left(\gamma(r)\left(b_{i}\right)\right)
$$

with the help of the basis $\left\{b_{i}\right\}$ of $H$ and the dual basis $\left\{\beta^{i}\right\}$ of $\hat{H}$.

$u \in V_{0}$ is normalized if

$$
\hat{S}^{-1}\left(\rho_{(2)}\right) u \rho_{(1)}=1_{\mathcal{D}\left(K_{c o p}^{o p}\right)^{o p}}
$$

holds in the double $\mathcal{D}\left(K_{c o p}^{o p}\right)^{o p}$.

\section{Appendix: Yetter-Drinfel'd modules over WHA's and Drinfel'd doubles}

For the convenience of the reader we give here the generalization of the double construction due to Drinfel'd [9] and of the corresponding theory of Yetter-Drinfel'd modules [16, 14] to WHA's.

Definition 6.1 [1] Let $H$ be a finite dimensional WHA over the field $k$. Its Drinfel'd double $\mathcal{D}(H)$ is the WHA defined below:

As a k-space $\mathcal{D}(H)$ is an amalgamated tensor product $H_{H^{L} \equiv \hat{H}^{R}} \otimes_{H^{R} \equiv \hat{H}^{L}} \hat{H}$ with the amalgamation relations $a^{R} \otimes \hat{\mathbb{1}} \equiv \mathbb{1} \otimes\left(\hat{\mathbb{1}}<a^{R}\right) ;\left(a^{L} \otimes \hat{\mathbb{1}}\right) \equiv \mathbb{1} \otimes\left(a^{L} \rightarrow \hat{\mathbb{1}}\right)$ for $a^{L} \in H^{L}, a^{R} \in H^{R}$. Denote by $\mathcal{D}(a) \mathcal{D}(\phi)$ the image of $H \otimes \hat{H} \ni a \otimes \phi$ under the amalgamation and $\mathcal{D}(a) \equiv \mathcal{D}(a) \mathcal{D}(\hat{\mathbb{1}}), \mathcal{D}(\phi) \equiv \mathcal{D}(\mathbb{1}) \mathcal{D}(\phi)$.

The algebra structure is defined by

$$
\begin{aligned}
\mathcal{D}(a) \mathcal{D}(b) & =\mathcal{D}(a b) \\
\mathcal{D}(\phi) \mathcal{D}(\psi) & =\mathcal{D}(\phi \psi) \\
\mathcal{D}(\phi) \mathcal{D}(a) & =\mathcal{D}\left(a_{(2)}\right) \mathcal{D}\left(\phi_{(2)}\right)\left\langle\phi_{(1)} \mid a_{(3)}\right\rangle\left\langle\phi_{(3)} \mid S^{-1}\left(a_{(1)}\right)\right\rangle
\end{aligned}
$$

One checks that (6.1) is compatible with the amalgamation relations and makes $\mathcal{D}(H)$ an associative algebra with unit $\mathcal{D}(\mathbb{1}) \equiv \mathcal{D}(\hat{\mathbb{1}})$. 
The colagebra structure is given by

$$
\begin{aligned}
\Delta_{\mathcal{D}}(\mathcal{D}(a) \mathcal{D}(\phi)) & =\mathcal{D}\left(a_{(1)}\right) \mathcal{D}\left(\phi_{(2)}\right) \otimes \mathcal{D}\left(a_{(2)}\right) \mathcal{D}\left(\phi_{(1)}\right) \\
\varepsilon_{\mathcal{D}}(\mathcal{D}(a) \mathcal{D}(\phi)) & =\varepsilon(a(\phi \rightarrow \mathbb{1})) \equiv \hat{\varepsilon}((\hat{\mathbb{1}} \leftarrow a) \phi) .
\end{aligned}
$$

One checks that (6.9) makes $\mathcal{D}(H)$ a WBA. Finally the antipode is

$$
S_{\mathcal{D}}(\mathcal{D}(a) \mathcal{D}(\phi))=\mathcal{D}\left(\hat{S}^{-1}(\phi)\right) \mathcal{D}(S(a))
$$

making $\mathcal{D}(H)$ a WHA.

Definition 6.2 Let $H$ be a WBA over the field $k$. The $k$-space $M$ is a right Yetter-Drinfel'd module over $H$ if it is a non-degenerate right $H$-module and a non-degenerate left $H$ comodule s.t.

$$
\begin{aligned}
m_{<-1>} a_{(1)} \otimes m_{<0>} \cdot a_{(2)} & =a_{(2)}\left(m \cdot a_{(1)}\right)_{<-1>} \otimes\left(m \cdot a_{(1)}\right)_{<0>} \\
m_{<-1>} \mathbb{1}_{(1)} \otimes m_{<0>} \cdot \mathbb{1}_{(2)} & =m_{<-1>} \otimes m_{<0>}
\end{aligned}
$$

for all $m \in M, a \in A$.

Notice that if $H$ is also a WHA then (6.4) can be replaced by the single relation

$$
(m \cdot a)_{<-1>} \otimes(m \cdot a)_{<0>}=S^{-1}\left(a_{(3)}\right) m_{<-1>} a_{(1)} \otimes m_{<0>} \cdot a_{(2)} .
$$

By the category $\mathcal{Y} \mathcal{D}(H)$ we mean the category with objects the finite dimensional right Yetter-Drinfel'd modules over $H$ and arrows $T: M \rightarrow M^{\prime}$ intertwining both the $H$-module and the $H$-comodule structures of $M$ and $M^{\prime}$.

If $H$ is a finite dimensional WHA then by our Proposition 4.2 and Example 4. the category $\mathcal{Y} \mathcal{D}(H)$ is equivalent to the category of the right modules over the WHA $\mathcal{D}(H)$ hence carries (among others) a monoidal structure [1, 2]. It is not so obvious however that it is true for any WBA $H$ :

Proposition 6.3 Let $H$ be a WBA over the field $k$. Then the category $\mathcal{Y} \mathcal{D}(H)$ has a monoidal structure.

Proof: Our proof is constructive. For two objects $M, N$ and arrows $T, S$ of $\mathcal{Y} \mathcal{D}(H)$ let

$$
\begin{aligned}
& M \times N \quad:=M \cdot \mathbb{1}_{(1)} \otimes N \cdot \mathbb{1}_{(2)} \quad \text { as a } k-\text { space } \\
&(m \otimes n) \cdot a:=m \cdot a_{(1)} \otimes n \cdot a_{(2)} \\
& \rho_{M \times N}(m \otimes n):=n_{<-1>} m_{<-1>} \mathbb{1}_{(1)} \otimes m_{<0>} \cdot \mathbb{1}_{(2)} \otimes n_{<0>} \cdot \mathbb{1}_{(3)} \\
& T \times S \quad:=(T \otimes S) \circ \Delta(\mathbb{1})
\end{aligned}
$$

with $m \otimes n \in M \times N, a \in A$. The monoidal unit is

$$
\begin{array}{ll}
H^{L} \text { as a } k-\text { space } & a^{L} \cdot h:=\mathbb{1}_{(2)} \varepsilon\left(a^{L} h \mathbb{1}_{(1)}\right) \\
& \rho_{H^{L}}:=\left.\Delta\right|_{H^{L}}
\end{array}
$$


for $a^{L} \in H^{L}, h \in H$.

The reader may check using some WBA calculus that all $M \times N$ and $H^{L}$ are Yetter-Drinfel'd modules over $H$ if $M$ and $N$ are.

In order to prove that $H^{L}$ is a monoidal unit for the category $\mathcal{Y} \mathcal{D}(H)$ one has to construct the invertible intertwiners $u_{M}^{L} \in\left(M, H^{L} \times M\right)_{\mathcal{Y} \mathcal{D}(H)}, u_{M}^{R} \in(M, M \times$ $\left.H^{L}\right)_{\mathcal{Y D}(H)}$ satisfying the triangle identities [11] and being natural in $M$. They are as follows:

$$
\begin{aligned}
& u_{M}^{L}(m)=\mathbb{1}_{(2)} \otimes m \cdot \Pi^{L}\left(\mathbb{1}_{(1)}\right) \\
& u_{M}^{R}(m)=m \cdot \mathbb{1}_{(1)} \otimes \mathbb{1}_{(2)} .
\end{aligned}
$$

for all $m \in M$ and all objects $M$ of $\mathcal{Y} \mathcal{D}(H)$.

\section{References}

[1] G. Böhm, K. Szlachányi, Lett. Math. Phys 35 (1996) p.437

[2] G. Böhm, F. Nill, K. Szlachányi, 'Weak Hopf Algebras I: Integral Theory and $C^{*}$-structure' math.QA/9805116 to appear in J. Algebra

[3] G. Böhm, F. Nill, K. Szlachányi, 'Weak Hopf Algebras II: Representation Theory, Dimensions, and the Markov Trace' in preparation

[4] C. Caenepeel, G. Militaru, S. Zhu, Israel J. Math 100 (1997) p.221

[5] C. Caenepeel, G. Militaru, S. Zhu, Trans AMS 349 (1997) p.4311

[6] C. Caenepeel, G. Militaru, S. Zhu, J. Algebra 187 (1997) p.388; C. Caenepeel, B. Ion, G. Militaru. S. Zhu 'Separable functors for the category of Doi-Hopf modules. Applications' math.QA/9809021

[7] Y. Doi, J. Algebra 153 (1992) p.373

[8] Y. Doi, Comm. Algebra 13 (1985) p.2137

[9] V. G. Drinfel'd, Proc. Int. Congr. Math. Berkeley (1986) p.798

[10] M. Koppinen, 'Variations of the smash product with applications to group graded rings' preprint 1991

[11] S. McLane, 'Categories for the working mathematician' Springer 1971

[12] F. Nill, 'Axioms for Weak Bialgebras' math.QA/9805104

[13] F. Nill, K. Szlachányi, H-W Wiesbrock, 'Weak Hopf Algebras and Reducible Jones Inclusions of Depth 2' math.QA/9806130

[14] D. Radford, J. Towber, J. Pure and Appl. Algebra 87 (1993) p.259

[15] M. E. Sweedler, 'Hopf algebras' Benjamin 1969

[16] D. N. Yetter, Math. Proc. Cambridge Phil. Soc. 108 (1990) p.261 